\title{
Ga3 subunit Thga3 positively regulates conidiation, mycoparasitism, chitinase activity, and hydrophobicity of Trichoderma harzianum
}

Jie Ding ${ }^{1}$, Jie Mei ${ }^{1}$, Pei Huang ${ }^{1}$, Ying Tian ${ }^{1,2}$, Yao Liang ${ }^{1}$, Xiliang Jiang ${ }^{1 *}$ and Mei Li ${ }^{1 *}$ (D)

\begin{abstract}
Heterotrimeric G-proteins are key elements of signal transduction pathways, which participate in regulating multiple biological processes in fungi including growth, conidiation, antagonism, and mycoparasitism. Among G protein subunits, Ga3 showed diverse regulatory functions in different fungi. In this study, we cloned a Ga3 subunit coding gene thga3 from T. harzianum Th33 that can antagonize Rhizoctonia solani and some other plant pathogenic fungi. A thga3 deletion strain $\triangle$ thga 3 was generated using the double-crossover homologous recombination strategy, and Rthga3 was generated by transforming thga3-expressing vector into the protoplasts of $\triangle$ thga3 by the $\mathrm{PEG} / \mathrm{CaCl}_{2}-$ mediated method. The biological characteristics of wild-type Th33, $\Delta$ thga3 and Rthga3 were evaluated. Compared with wild-type Th33, $\Delta$ thga3 showed 15\%,94\%, and 23\% decrease in hyphal growth, conidia yield, and chitinase activity, respectively, and $\Delta$ thga3 showed lower antagonistic and mycoparasitism abilities, while there were no significant differences between wild-type Th33 and Rthga3. The hyphal surface hydrophobicity of $\Delta$ thga3 significantly decreased compared with those of the wild-type Th33 and Rthga3. qRT-PCR analysis revealed that transcript abundance of the hydrophobin gene (tha_09745) of $\triangle$ thga3 decreased by $80 \%$ compared with that of wild-type Th33 and Rthga3. The results showed that thga3 positively regulates the growth, conidiation, hydrophobicity, chitinase activities, and mycoparasitism of Th33 towards R. solani. We hence deduced that the expression level of Tha_09745 is correlated to the hyphal hydrophobicity of Th33 and therefore affects the other biological characteristics of Th33. The findings of this report provide a foundation for elucidating the G-protein signal regulatory mechanisms of fungi.
\end{abstract}

Keywords: Thga3, Trichoderma harzianum, Conidiation, Mycoparasitism, Chitinase, Hydrophobicity

\section{Introduction}

Trichoderma spp. are widely used as biocontrol agents. Understanding their genetic regulation of biocontrol activities is beneficial to their genetic improvement and application as biofungicides. The biocontrol effects of Trichoderma preparations against plant pathogenic microorganisms are affected by a variety of factors, including development stage, environmental temperature, humidity, light, and nutrition. The current

\footnotetext{
*Correspondence: jiangxiliang@caas.cn; limei@caas.cn

${ }^{1}$ Institute of Plant Protection, Chinese Academy of Agricultural Sciences, No. 2 West Yuanmingyuan Rd., Haidian District, Beijing 100193, China Full list of author information is available at the end of the article
}

understanding of how Trichoderma senses external signals, transmits the external signals to the cells, and regulates growth and development is limited. G proteins play key roles in regulating the growth, development, reproduction, pathogenesis, and secondary metabolite biosynthesis in filamentous fungi (Nogueira et al. 2015; Lei et al. 2019). Heterotrimeric G-protein complexes consist of alpha $(\alpha)$, beta $(\beta)$, and gamma $(\gamma)$ subunits. Most filamentous fungi have three $G \alpha$ subu-

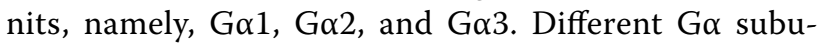
nits regulate different biological processes in fungi (Lei et al. 2019). Ga1 subunits are found more frequently and regulate vegetative growth, conidiation, and mycoparasitic responses in fungi (Rocha-Ramírez et al. 2002; 
Sun et al. 2016; Reithner et al. 2005). The function of $\mathrm{G} \alpha 2$ in fungi is rarely reported (Lei et al. 2019), and $\mathrm{G} \alpha 3$ subunits possess different regulatory functions, the understanding of which is based on studies on $\mathrm{G \alpha 3}$ subunits from Trichoderma spp. (Schmoll et al. 2009; Susanne et al. 2005), Penicillium spp. (García-Rico et al. 2017; Hu et al. 2013), Valsa mali (Song et al. 2017), and Fusarium spp. (Yu et al. 2008; Guo et al. 2016). These regulate the growth, conidiation, cellulase, and chitinase activities of fungi. Several Ga3 subunits from various Trichoderma strains exhibit relatively different functions. For example, GNA3 (Go3) from T. reesei (do Nascimento et al. 2009) and GNA3 (Ga3) in T. reesei (Schmoll et al. 2009) are related to cellulase activity, while Tga3 $(\mathrm{G} \alpha 3)$ in $T$. viride affects chitinase gene expression (Susanne et al. 2005) and Tga3 (Go3) in $T$.

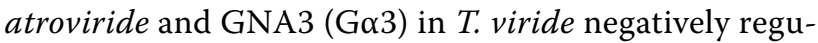
late conidiation. Light seems to play a role in regulating functions of Ga3 subunits in Trichoderma (Schmoll et al. 2009; Susanne et al. 2005). Studying the functions of Ga3 subunits of different Trichoderma strains may further clarify their functions and regulatory mechanisms.

This study cloned a Th33 Ga3 gene, thga3, that can antagonize multiple plant pathogenic fungi, including Rhizoctonia solani, Fusarium spp. and Phytophthora spp. In addition, we observed that the function of this gene differs from that of previously reported Ga3. It positively regulates the growth, conidiation, and chitinase activities as well as the mycoparasitism ability of Th33 on $R$. solani. Both hyphal hydrophobicity and the expression of a type II hydrophobin gene Tha_09745 in Th33 are positively regulated by Thga3. These findings indicate that the hydrophobicity of Th33 is correlated to the expression of Tha_09745 and therefore influences the biological characteristics of Th33.

\section{Materials and methods}

\section{Strains and culture conditions}

The strains used in this study include wild-type T. harzianum Th33 (CGMCC No. 19906), mutant $\Delta$ thga3 (thga3 deletion strain), mutant Rthga3 (thga3 complemented strain), and pathogenic fungi $R$. solani (ACCC No. 36124). For microscopic observation and mycelial biomass determination, strains were inoculated into potato dextrose agar (PDA) and incubated at $25^{\circ} \mathrm{C}$ for 4 days. Escherichia coli Trans1-T1 competent cells were purchased from TransGen (TransGen Biotech, Beijing, China) and used for cloning and propagation of plasmids. Hygromycin B (hyg, $200 \mathrm{mg} / \mathrm{mL}$ ) was added to PDA to screen $\Delta$ thga3, and geneticin (G418 sulfate, $100 \mathrm{mg} / \mathrm{mL}$ ) was used for screening Rthga3.

\section{Deletion of thga 3 in T. harzianum Th33}

The Ga3 gene thga3 of T. harzianum Th33 was cloned from the Th33 genomic DNA (GenBank Accession Number PRJNA272949) (Sun et al. 2016). The thga3 deletion strain $\Delta$ thga 3 was generated by the double-crossover homologous recombination strategy. The deletion strategy is shown in Fig. 1a using the primers listed in Additional file 1: Table S1. Plasmid pKH-KO (Wang et al. 2014) was used as a transformation vector containing two uracil-specific excision reagent (USER) cloning sites, USC1 and USC2, on either side of the hygromycin B gene hyg (Wang et al. 2014). The $5^{\prime}$ flanking region $(1,110 \mathrm{bp})$ of the thga 3 coding sequence was cloned from genomic DNA of Th33 and cloned into the USC2 sites of HindIII/XhoI-digested pKH-KO using Clontech In-Fusion ${ }^{\circledR} \mathrm{HD}$ Cloning Kit (TaKaRa). The $3^{\prime}$ flanking region (1036 bp) was cloned into the USC1 sites of SpeI/ EcoRI-digested $\mathrm{pKH}-\mathrm{KO}$ in the same orientation as that of the $5^{\prime}$ flanking region to generate the Thga3 disruption vector $\mathrm{pKH}-\mathrm{KO}$-thga3. The $\mathrm{PEG} / \mathrm{CaCl}_{2}$-mediated method (Aragona and Valente, 2015) was used to generate the $\Delta$ thga 3 by transforming $\mathrm{pKH}-\mathrm{KO}$-thga 3 into the protoplasts of wild-type Th33, and the genotypes of $\Delta$ thga 3 mutants were confirmed by amplifying internal fragments of thga3 (no PCR product generated), and the hyg fragment (PCR product was $1221 \mathrm{bp}$ in size).

\section{Complementation of thga3}

To generate the complemented strain of the thga3 deletion mutant, a thga3 complementation cassette containing the promoter PgpdA, terminator TtrpC, and the coding region of thga 3 was constructed. PgpdA and TtrpC fragments were amplified from the plasmid pAN71. The TtrpC fragment was first fused to the genome of wild-type Th33 and ligated with thga 3 to produce chimeras using gene splicing by overlap extension (SOE) (Shevchuk et al. 2004). The derived chimeras were used as a template for amplifying the fragment containing thga 3 and $\operatorname{TtrpC}$ sequences, which was then fused with the PgpdA using SOE and generated the thga 3 complementation cassette, which was then cloned into SpeI/EcoRIdigested pKH-KO-G using a Clontech In-Fusion ${ }^{\circledR} \mathrm{HD}$ cloning kit. The resulting complementing vector was designated as pKH-KO-Gthga3 (Fig. 1b). pKH-KO-Gthga3 was then transferred into $\Delta$ thga 3 protoplasts. The thga 3 complemented strain Rthga3 was screened by G418 resistance $(100 \mathrm{mg} / \mathrm{mL})$, and the genotypes were confirmed by PCR amplifying internal fragments of thga3 and neo (G 418 resistance gene).

Reverse-transcription polymerase chain reaction (RTPCR) was used to confirm the transcription of thga 3 in wild-type Th33 and the mutants. Total RNA was isolated 


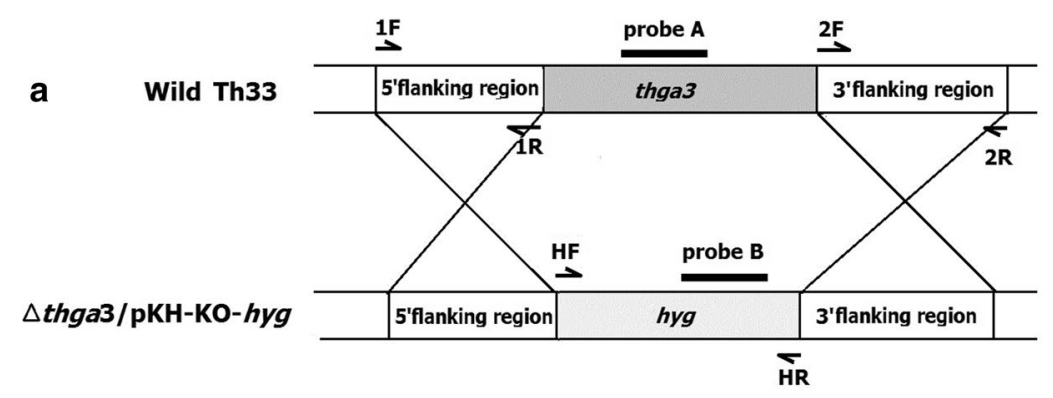

b

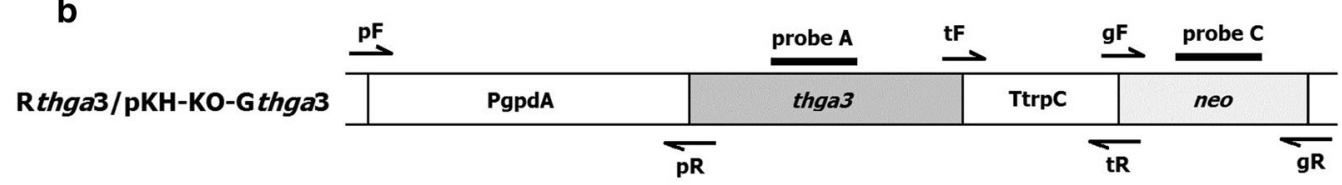

C
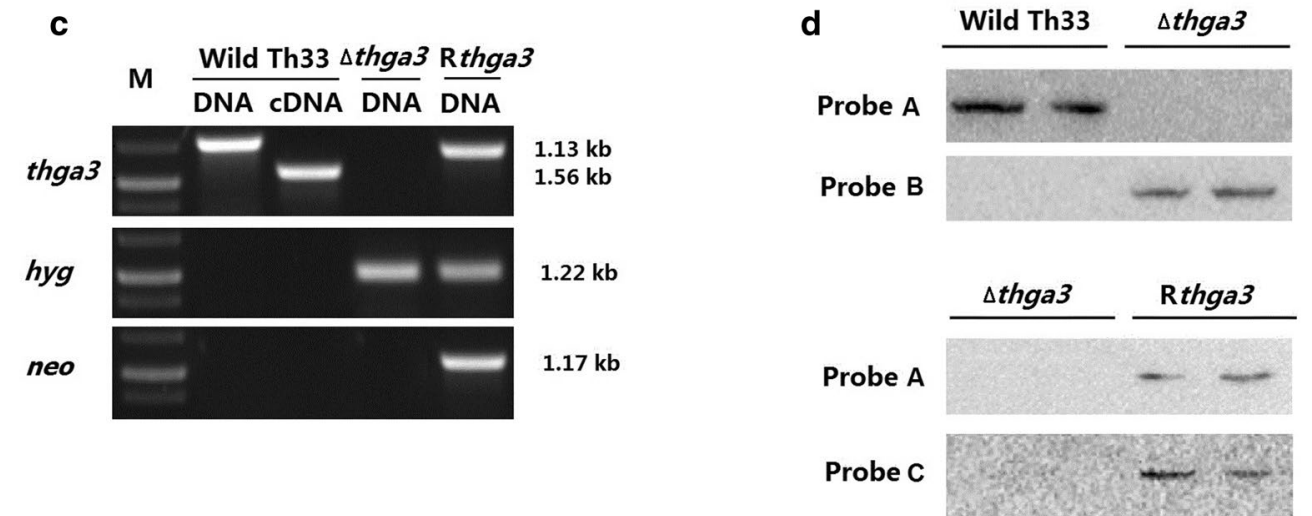

Fig. 1 Construction of thga3 deletion and complemented mutants. a Thga3 gene deleting strategy. $\mathbf{b}$ Thga3 gene complementing vector pKH-KO-Gthga3. c PCR and RT-PCR identification of thga3, hyg, and neo genes in $\triangle$ thga3 and Rthga3. M, 250-bp ladder. PCR templates were genomic DNA and cDNA of wild-type Th33, genomic DNAs of $\triangle$ thga3 and Rthga3. d Southern hybridization analysis. Probe A. Thga3 ORF; Probe B. Hyg gene; ProbeC. Neo gene. DNAs of wild-type Th33, $\triangle$ thga3 and Rthga3 were digested by EcoRl/Xhol

using TransZol Up Plus RNA. cDNA was prepared from total RNA using FastQuant RT kit (With gDnase) (Tiangen). R-Taq polymerase (TaKaRa) was used for PCR amplification of thga3 from cDNAs.

\section{Southern blotting}

Southern hybridization was performed using a DIGHigh prime DNA labeling and detection starter kit II (Roche, Germany) according to the manufacturer's protocol. Fragments of thga3 [Probe A, 371 bp], hyg [selective marker gene, probe B (426 bp)], and neo [selective marker gene, probe $C$ ] were amplified for use as probes. DNAs of wild-type Th33 and mutants $\Delta$ thga 3 and Rthga 3 were digested by EcoRI/Xhol. The primers used in this assay are listed in Additional file 1: Table S1.

\section{Growth, conidiation, and hydrophobicity}

Wild-type Th33, $\Delta$ thga3, and Rthga3 were respectively inoculated at the center of PDA plates and cultured for 5 days at $25{ }^{\circ} \mathrm{C}$. The colony morphology of each strain was monitored, and radial hyphal growth rates were measured daily. Twenty culture discs were collected from each culture using a cork borer (5-mm diameter) after 6 days of incubation and placed into $10 \mathrm{~mL}$ of sterile distilled water and vortexed. The number of spores was counted under microscope with a hemacytometer.

The hydrophobicity of hyphal surface of colonies was tested by dropping $15 \mu \mathrm{L}$ of $0.5 \%$ aqueous aniline blue on fully grown ( $6 \mathrm{~d}$ ) colonies of wild-type Th33, $\Delta$ thga3, and Rthga3, and observing the disappearance of the water or dye over an 8-h period (Mukherjee and Kenerley 2010). 


\section{Antagonism and mycoparasitism assays}

A dual culture technique (Dennis and Webster, 1971) was used for assessing the antagonistic activities of wild-type Th33 and its mutants against $R$. solani. Five-millimeter discs of Trichoderma and $R$. solani from 3-day-old cultures were placed in a PDA plate $(90-\mathrm{mm}$ diameter) $50 \mathrm{~mm}$ apart. A control plate was maintained with $R$. solani alone and incubated at room temperature $\left(25^{\circ} \mathrm{C}\right)$. Growth rate and colony morphology were assessed daily for six days. The percentage of growth inhibition was calculated using the equation $\mathrm{RI}=100 \times\left(\mathrm{R}_{2}-\mathrm{R}_{1}\right) / \mathrm{R}_{2}(\mathrm{Li}$ et al. 2019), where RI is the percentage of reduction in mycelial growth, $\mathrm{R}_{1}$ is the mycelial growth of $R$. solani in dual plates, and $R_{2}$ is the mycelial growth of $R$. solani in the control. Three replicates were prepared for each treatment.

The slide culture method was used to investigate mycoparasitism of Trichoderma against $R$. solani. A glass microscope slide covered with a thin layer of $0.8 \%$ water agar (WA) was inoculated with 5-mm diameter mycelial discs of T. harzianum and $R$. solani, $10 \mathrm{~mm}$ apart from each other, and cultured at $25^{\circ} \mathrm{C}$. The regions where the hyphae of the two strains met were periodically observed under a light microscope as described previously (Jiang et al. 2016).

\section{Chitinase assays}

The chitinase activities of Trichoderma strains were tested on colloidal chitin agar and liquid induced medium. The composition of colloidal chitin agar was $\mathrm{MgSO}_{4} \cdot 7 \mathrm{H}_{2} \mathrm{O} \quad(3 \mathrm{~g} / \mathrm{L}), \quad\left(\mathrm{NH}_{4}\right)_{2} \mathrm{SO}_{4}(3 \mathrm{~g} / \mathrm{L}), \quad \mathrm{KH}_{2} \mathrm{PO}_{4}$ $(2 \mathrm{~g} / \mathrm{L})$, citric acid $(1 \mathrm{~g} / \mathrm{L})$, Tween-80 $(200 \mu \mathrm{L} / \mathrm{L})$, agar (15 g/L), and 5\% colloidal chitin (5.25 g/L), pH 4.7. Colloidal chitin was prepared by the method of Sakai et al. (1998). Wild-type Th33, $\Delta$ thga3, and Rthga3 were inoculated independently at the center of colloidal chitin agar plates and cultured for 5 days at $25^{\circ} \mathrm{C}$. The colony growth and culture medium color changes were observed; the chitinase activity was proportional to the increasingly dark color of the medium. The chitinase activities of the wild type Th33, $\Delta$ thga3, and Rthga 3 in culture filtrates were determined as described elsewhere (Fernandes et al. 2013). The absorbance was measured at a wavelength of $585 \mathrm{~nm}$, and one unit of enzyme (U) was defined as the amount of enzyme necessary to produce $1 \mathrm{mg}$ of $N$-acetylglucosaminidase per gram dry weight hyphae in $1 \mathrm{~h}$. The test was repeated three times.

\section{Quantitative real-time PCR for detection of hydrophobin gene expression}

Total RNA extraction and cDNA synthesis were performed as earlier described (Song et al. 2017). Primers were designed by Prime Express Software v2.0, and are listed in Additional file 2: Table S2. Ubiquitin-conjugating enzyme (UCE) gene (KX686115) was used for internal standard. Quantitative real-time PCR was performed on an ViiA 7 Real time system (ABI) system using a QuantiFast SYBR Green PCR Kit (400) (Qiagen). Three parallel experiments were performed of each sample in a total volume of $16 \mu \mathrm{L}$. The instrument was programmed for $2 \mathrm{~min}$ at $95^{\circ} \mathrm{C}$, followed by 40 cycles of $10 \mathrm{~s}$ at $94{ }^{\circ} \mathrm{C}$, $10 \mathrm{~s}$ at $60{ }^{\circ} \mathrm{C}$, and $40 \mathrm{~s}$ at $72{ }^{\circ} \mathrm{C}$.

\section{Statistical analysis}

The statistical software SAS 8.0 (SAS Institute, Inc., Cary, NC, USA) was used for ANOVA. Normality assumptions of the measured variables were checked, and no data transformation was required. Duncan's multiple range tests were used to compare the means obtained after each experiment. A value of $\mathrm{P}<0.05$ was considered statistically significant. The software Origin 8 was used for drawing.

\section{Results \\ Construction and validation of thga 3 deleted and complemented mutants}

The Thga3 gene was cloned from wild-type Th33 genome, which contains six exons and five introns and encoding 355 amino acids (GenBank Acc. No. KY937956). There was a single copy of thga3 in the wild-type Th33 genome (data not shown). The thga3 single-deleted strain $\Delta$ thga3 with resistance to hygromycin B and thga3 complemented mutant Rthga3 with resistance to G418 were obtained (Fig. 1c, d). For the $\Delta$ thga 3 strain, the PCR analysis confirmed the existence of the hyg sequence and the absence of the thra3 sequence. Southern hybridization showed a single copy of the hyg sequence in $\Delta$ thga3. For the Rthga3 strain, PCR analysis confirmed the existence of thga3, hyg, and neo sequences. Southern hybridization showed a single copy of the thga3 and neo sequence in the Rthga3 (Fig. 1c, d). The primers used for PCR, RT$\mathrm{PCR}$, and the probes for southern hybridization are listed in Additional file 1: Table S1.

\section{Morphology, growth, and conidiation}

When incubated on PDA medium, there were no significant differences in colony morphology, growth, and conidiation between wild-type Th33 and Rthga3. $\Delta$ Thga 3 showed a $15 \%$ reduction $(\mathrm{P}<0.001)$ in growth rate compared with wild-type Th33 $(1.4 \mathrm{~cm} /$ day linear growth, compared with wild-type Th33 growth of $1.7 \mathrm{~cm} /$ day), when grown on PDA plates at $25{ }^{\circ} \mathrm{C}$ for $48 \mathrm{~h}$ (Fig. 2a), and the formation of aerial hyphae and conidia significantly decreased compared with wild-type Th33. The conidia yield of wild-type Th33, $\Delta$ thga3, and Rthga3 was $1.26 \pm 0.17 \times 10^{7}$ spores $/ \mathrm{cm}^{2}$, 

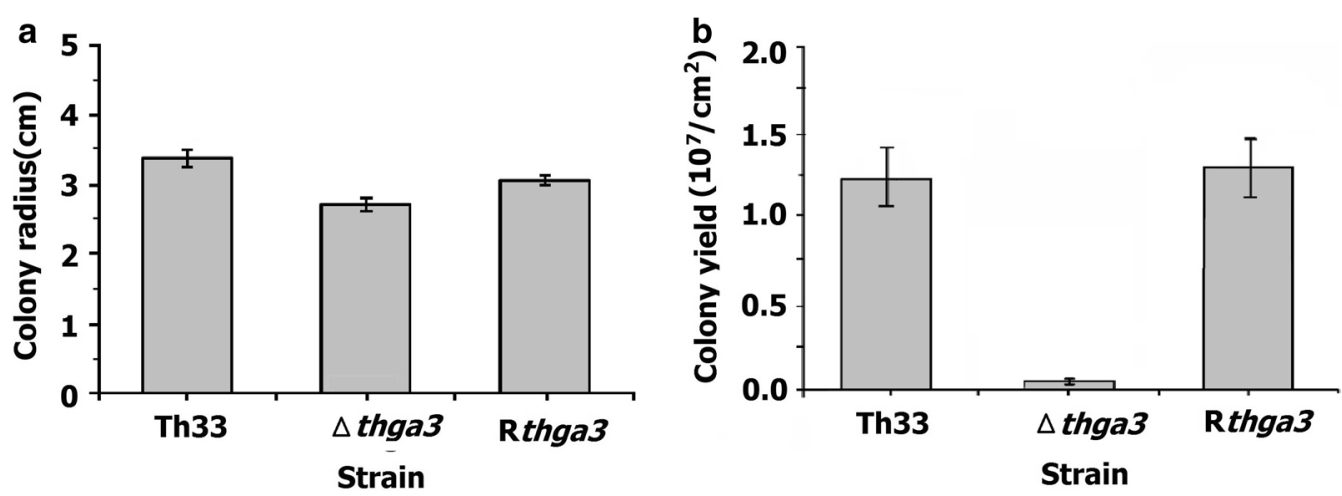

Fig. 2 Growth and conidiation of the T. harzianum wild-type Th33, $\Delta$ thga3, and Rthga3. a Growth of wild-type Th33, $\triangle$ thga3, and Rthga3 on PDA for $48 \mathrm{~h}$ at $25^{\circ} \mathrm{C}$. $\mathbf{b}$ Conidia yield of wild-type Th33, $\triangle$ thga3, and Rthga3, on PDA for $6 \mathrm{~d}$ at $25^{\circ} \mathrm{C}$. Error bars represent the SD of three determinations for two independent experiments

$7.7 \pm 0.58 \times 10^{5}$ spores $/ \mathrm{cm}^{2}$, and $1.32 \pm 0.01 \times 10^{7}$ spores $/ \mathrm{cm}^{2}$, respectively, after culturing on PDA for 6 d. $\Delta$ Thga3 conidia yield was approximately $94 \%$ lower $(\mathrm{P}<0.001)$ than that of wild-type Th33 and Rthga3 $(\mathrm{P}<0.0001)$ (Fig. 2b).

\section{Antagonistic activities against $R$. solani}

When confront cultured with $R$. solani, all the wild-type Th33, $\Delta$ thga3, and Rthga 3 could overgrow the colony of $R$. solani and further produce clusters of spores with different inhibitory effects (Fig. 3a). The growth inhibition ratios of wild-type Th33 and Rthga 3 against $R$. solani were $83.83 \pm 1.01 \%$ and $84.79 \pm 0.90 \%$, respectively, which a

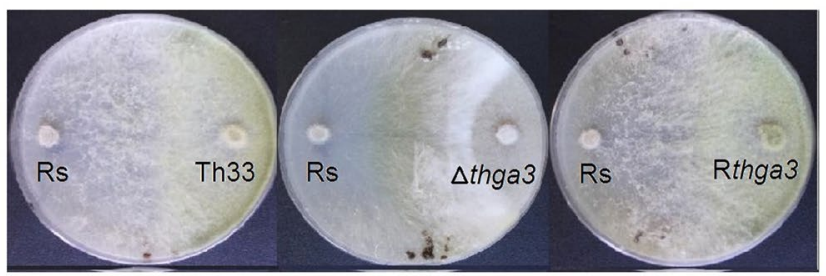

C

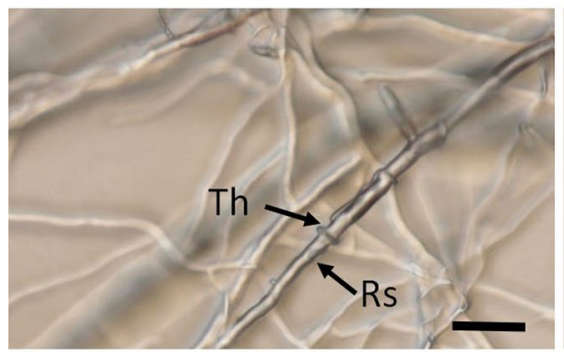

Th33

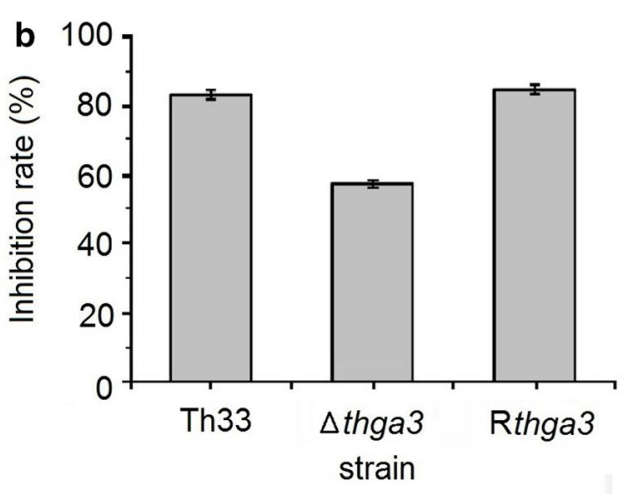

Fig. 3 Antagonistic activities of the T. harzianum wild-type Th33, $\Delta$ thga3, and Rthga3 against R. solani (Rs). a Confrontation assay of wild-type Th33, $\triangle$ thga3, and Rthga 3 against Rs. Cultures were grown on PDA plates for $10 \mathrm{~d}$ at $25^{\circ} \mathrm{C}$. $\mathbf{b}$ Inhibition ratio of the wild-type Th33, $\Delta$ thga3, and Rthga3 on the growth of Rs (grown on PDA plates for $6 \mathrm{~d}$ at $25^{\circ} \mathrm{C}$ ); error bars represent the SD of five determinations for two independent experiments. $\mathbf{c}$ Mycoparasitism of wild-type Th33, $\Delta$ thga3, and Rthga3 against Rs. For wild-type Th33 and Rthga3, the hyphae grew along and coiled the hyphae of Rs; for $\Delta$ thga3, no coiled growth of the hyphae to the Rs was observed. Bars $=40 \mu \mathrm{m}$ 
were significantly higher than $58.24 \pm 0.19 \%$ of $\Delta$ thga3 after confront culturing for $6 \mathrm{~d}(\mathrm{P}<0.0001)$. The inhibition rate of $\Delta$ thga 3 decreased by about $31 \%$ compared to those of wild-type Th33 and Rthga3. The hyphae of the wild-type Th33 and Rthga3 grew around the hyphae of $R$. solani (Fig. 3a, c), and part of the hyphae of $R$. solani underwent fragmentation. In $\Delta$ thga 3 , the hyphae of $\Delta$ thga 3 and $R$. solani grew independently and were not affected by each other, even when the hyphae of $\Delta$ thga 3 and $R$. solani came into contact, showing that $\Delta$ thga 3 lost its mycoparasitism ability against $R$. solani (Fig. 3b).

\section{Thga3 regulates the hyphal hydrophobicity} and the expression of the hydrophobin gene

Wild-type Th33 and Rthga 3 were highly hydrophobic, but $\Delta$ thga3 was hydrophilic (Fig. 4a). The transcript level of the hydrophobin gene (tha_09745) of $\Delta$ thga3 decreased by $80 \%$ compared with that of wild-type Th33 and Rthga 3 as detected by qRT-PCR (Fig. 4b), whereas no significant differential expression of the other five hydrophobin genes was observed in $\Delta$ thga 3 compared with wild-type Th33 (Additional file 3: Fig. S1). The results showed that both the hydrophobicity and expression of Tha_09745 were positively regulated by Thga3, and we deduced that the expression level of Tha_09745 is correlated to the hyphal hydrophobicity of Th33.

\section{Thga3 regulates chitinase activities}

When wild-type Th33, $\Delta$ thga3, and Rthga 3 were growing on chitinase-inducing medium, the medium turned to dark purple with different discoloration ranges 5 days later (Fig. 5a). The diameters of purple areas of wildtype Th33 $(4.6 \pm 0.3 \mathrm{~cm})$ and Rthga $3(4.4 \pm 0.2 \mathrm{~cm})$ were significantly greater than that of $\Delta$ thga3 $(3.6 \pm 0.2 \mathrm{~cm})$ (Fig. 5b). The corresponding activities of $\Delta$ thga3 ( $82 \pm 1.3$ $\mathrm{ug} / \mathrm{h} / \mathrm{mL}$ ) reduced by $23 \%$ compared with that of wildtype Th33 $(100 \pm 6.5 \mathrm{ug} / \mathrm{h} / \mathrm{mL})$ and Rthga3 $(110 \pm 5.1$ $\mathrm{ug} / \mathrm{h} / \mathrm{mL})$, showing that Thga3 positively regulated chitinase activity of Th33.

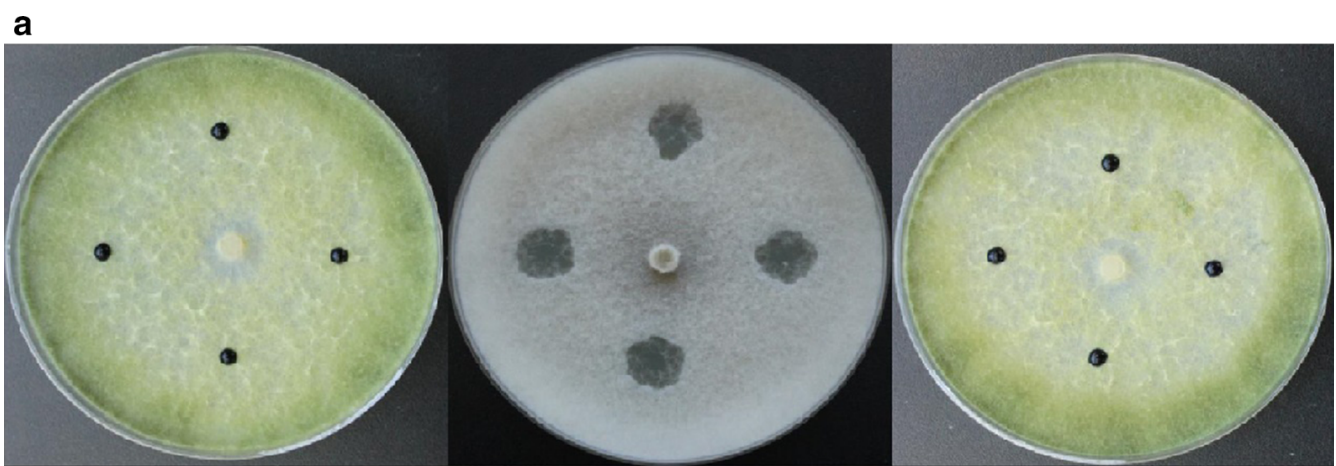

Th33

$\triangle$ thga3

Rthga3

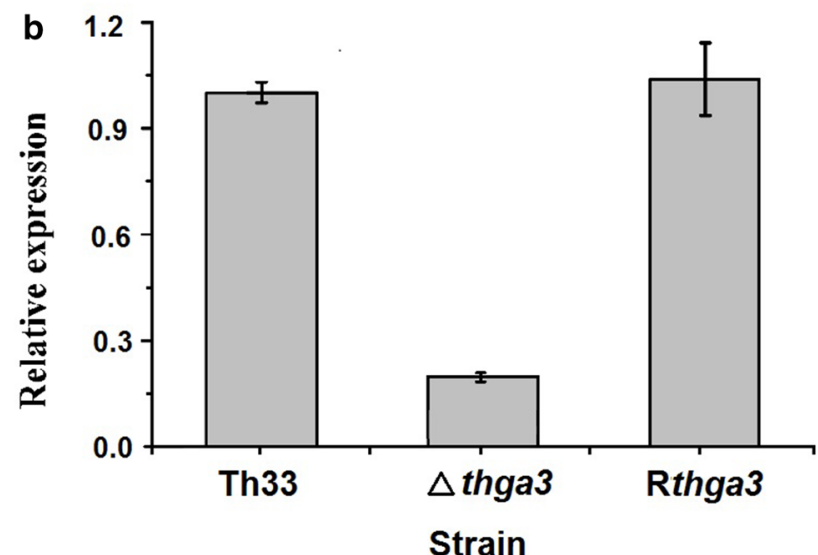

Fig. 4 Hydrophobicity and expression of hydrophobin gene Tha_09745 of wild-type T. harzianum Th33, $\Delta$ thga3, and Rthga3. a Hydrophobicity of wild-type T. harzianum Th33, $\Delta$ thga3, and Rthga3. Fifteen microliters of $0.5 \%$ aqueous aniline blue was spotted onto colonies and imaged after $8 \mathrm{~h}$. b Relative expression levels of Tha_09745 in wild-type Th33, $\Delta$ thga3, and Rthga3 grown on PDA plates for 7 days at $25^{\circ} \mathrm{C}$ (fold-changes in mRNA expression relative to that of reference gene UCE). Error bars represent the SD of three determinations from two independent experiments 

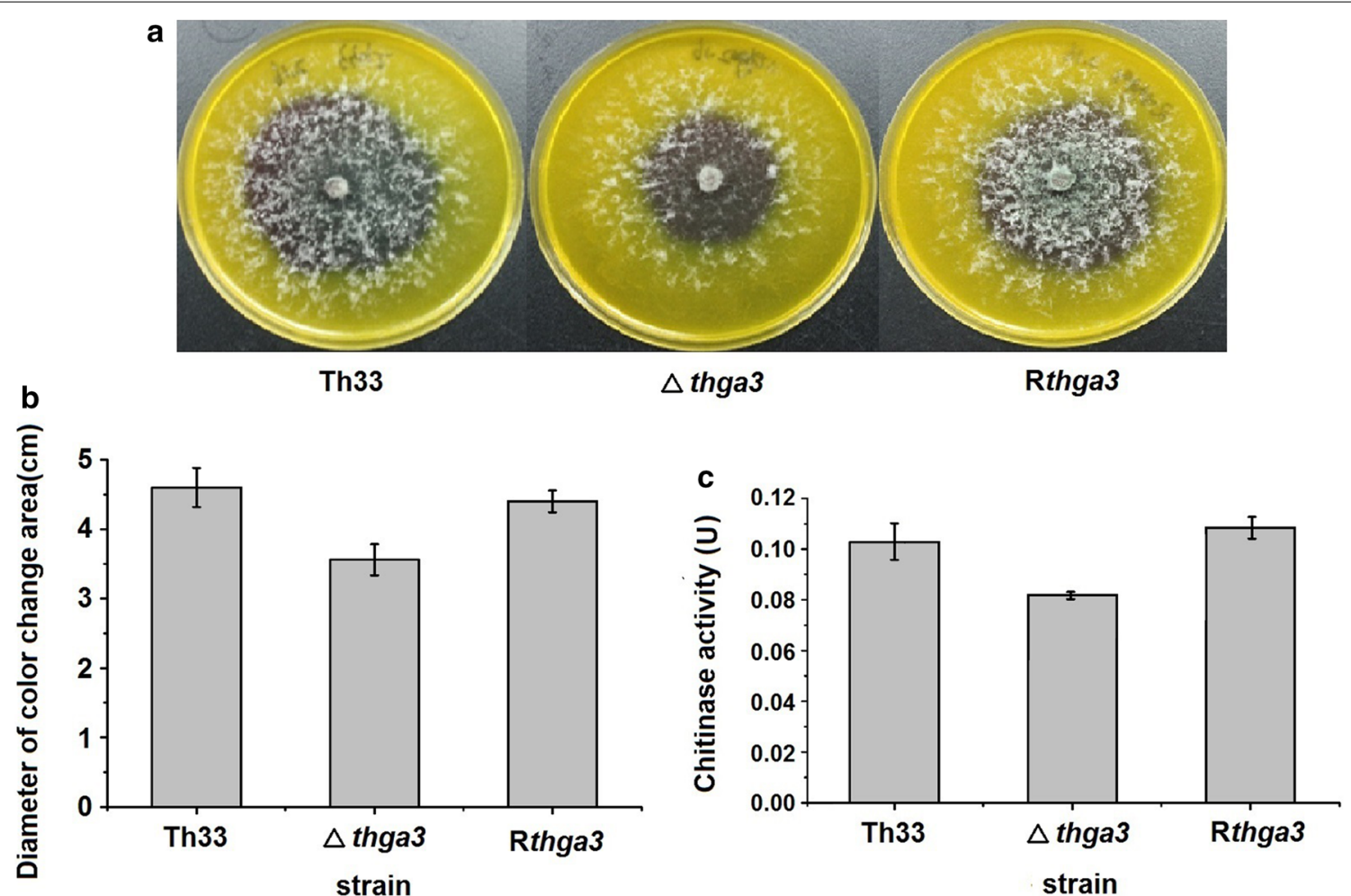

Fig. 5 Chitinase activities of wild-type Th33, $\Delta$ thga3, and Rthga3. a Discoloration of chitinase-inducing medium cultured with wild-type Th33, $\Delta$ thga3, and Rthga 3 at $28^{\circ} \mathrm{C} 5$ days later. $\mathbf{b}$ Diameter of discolored area of chitinase-inducing medium cultured with wild-type Th33, $\Delta$ thga3, and Rthga 3 at $28^{\circ} \mathrm{C} 5$ days later. $\mathbf{c}$ Chitinase activities of the culture filtrates of wild-type Th33, $\Delta$ thga3, and Rthga3. Error bars represent the SD of three determinations from two independent experiments

\section{Discussion}

Ga3 subunits in various fungi possess multiple different regulatory functions. Thga 3 in this study positively regulates the hyphal growth, which is similar with that of PGA3 (Ga3) in Penicillium camemberti (Hu et al. 2013), Gvm3 (Go3) in Valsa mali (Song et al. 2017), and GanB (Ga3) in Aspergillus nidulans (Chang et al. 2004). Furthermore, FGA3 (Ga3) in Fusarium oxysporum shows no influence on vegetative growth (Guo et al. 2016). Ga3 subunits in T. viride, T. atroviride, and Rhodospirillum sp. negatively regulate conidiation (Schmoll et al. 2009; Zeilinger et al. 2005), and GanB (Go3) in Aspergillus nidulans positively regulates conidial germination (Chang et al. 2004). Thga3 in this study positively regulates conidiation in Th33 and does not show influence on the conidial germination (data not shown).

GNA3 (Go3) from $T$. reesei participates in cellulase activity and antimicrobial peptide synthesis (do Nasci-

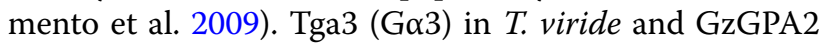
(Go3) in F. graminearum affect chitinase gene expression (Susanne et al. 2005), and PGA3 in P. decumbens regulates amylase and cellulase synthesis (Hu et al. 2013). In this study, the thga 3 knockout mutant shows a lower chitinase activity than those of the wild-type Th33 and thga3-complemented mutant Rthga3, meaning that it positively regulates the chitinase activity of Th33, which

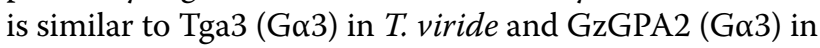
F. graminearum, but Thga3 shows no significant effect on cellulase and amylase activities (data not shown).

The regulatory functions of $\mathrm{G} \alpha 3$ in fungi are apparently affected by light. For example, Tga3 in T. atroviride shows hyper-conidiation in the dark with loss of tga3 (Susanne et al. 2005), and GNA3 in T. reesei itself is induced by light exposure and regulates the expression of cellulase that is in response to light (Schmoll et al. 2009). However, the regulatory functions of Thga3 on the growth, conidiation, and chitinase activity of Th33 in this study seem to have no correlation with light (data not shown).

Based on aforementioned comparison and analysis, we speculated that the role of Ga3 is diverse and complicated in different fungi species, which could influence growth, sporulation, chitinase and cellulase activities, as well as their responses to light in different strains. However, the reports concerning the function of Ga3 in fungi are still limited, and its regulation mechanism is not revealed yet. Therefore, study on the 
regulatory mechanisms of Ga3 will help us to uncover the difference of its function in different strains".

T. harzianum Th33 in this study is a biocontrol fungi used in controlling plant fungal diseases caused by $R$. solani, Sclerotium rolfsii, Sclerotinia sclerotiorum, $F$. oxysporum, and Pythium spp. (Sun et al. 2016). Deleting thga3 resulted in a significant decrease in antagonistic and mycoparasitic ability, which is similar to GzGPA2 in $F$. graminearum, wherein deletion of GzGPA2 caused reduced pathogenicity and increased chitin accumulation in the cell wall (Yu et al. 2008). Chitinase was reported to have antifungal activities (Lorito et al. 1993). We thus deduced that the reduced chitinase activities of $\Delta$ thga 3 reduces the antagonistic and mycoparasitic ability of Trichoderma against $R$. solani.

Hydrophobins are small cysteine-rich surface active proteins produced by fungi on the outer surface of cell walls. The roles of hydrophobins in surface hydrophobicity, conidiation, fruit body formation, recognition, and adhesion onto the host surface and virulence have been investigated (Dubey et al. 2014; Minenko et al. 2014; Zhang et al. 2011). In this study, wild-type Th33 and Rthga 3 are hydrophobic, whereas the hyphae of $\Delta$ thga3 is hydrophilic. The Th33 genome (GenBank Acc. No. PRJNA272949) harbors six hydrophobinencoding genes. We detected the expressions of all of the six hydrophobin genes in wild-type Th33, $\Delta$ thga3, and Rthga3 (data not shown). Only the expression of Tha_09745 significantly decreased in $\Delta$ thga3 compared with wild-type Th33 and Rthga3, and there was no significant difference between wild-type Th33 and Rthga3, which corresponded with the hydrophobicity phenotype of the strains. We deduced that the expression level of Tha_09745 is correlated to the hyphal hydrophobicity of Th33, and loss of hydrophobicity may affect the surface recognition and mycoparasitism of Th33 against $R$. solani, although this requires further investigation.

Both $\mathrm{G}$ proteins and hydrophobin in fungi have a variety of regulatory functions, and numerous studies on this property have been conducted (Huang et al. 2015; Espino-rammer et al. 2013; Khalesi et al. 2015). However, studies on the correlation between the G proteins and hydrophobin in fungi are limited, Segers and Nuss (2003) reported that $\mathrm{G} \alpha$ negatively regulated hydrophobin gene expression in the chestnut blight fungus Cryphonectria parasitica. In this study, we found for the first time that Go in Trichoderma positively regulates the expression of the hydrophobin gene and hyphal hydrophobicity, which is also correlated to the growth, conidiation, and mycoparasitism of Th33. Our findings may be used in future studies on the regulatory mechanism of the G signal system in fungi.

\section{Supplementary Information}

The online version contains supplementary material available at https://doi. org/10.1186/s13568-020-01162-9.

Additional file 1: Table S1. Primers used for deletion and complementation of Thga3.

Additional file 2: Table S2. Primers for qRT-PCR detection the expression of six hydrophobin genes and UCE (ubiquitin-conjugating enzyme) gene as reference gene in wild-type Th33 and thga3 deletion strain $\triangle$ thga3.

Additional file 3: Figure S1. The relative expression of the five hydrophobin genes in wild-type Th33 and $\Delta$ thga3.

\section{Acknowledgments}

We thank LetPub (https://www.letpub.com) for its linguistic assistance during the preparation of this manuscript.

\section{Authors' contributions}

Experimental design and planning were performed by ML. Experiments were executed and data was processed by JD, JM, PH, YT and YL. Data analysis was done by $\mathrm{ML}$ and $\mathrm{XJ}$. The article was written by ML. All authors read and approved the final manuscript.

\section{Funding}

This study was supported by the National Natural Science Foundation of China (Grant No. 31371983) and the National Key R\&D Program of China (2017YFD0200900).

\section{Availability of data and materials}

Not applicable.

Ethics approval and consent to participate

Not applicable.

\section{Consent for publication}

Not applicable.

\section{Competing interests}

The authors declare no competing interests.

\section{Author details}

${ }^{1}$ Institute of Plant Protection, Chinese Academy of Agricultural Sciences, No. 2 West Yuanmingyuan Rd., Haidian District, Beijing 100193, China. ${ }^{2}$ College of Plant Protection, Yunnan Agricultural University, National Key Laboratory for Conservation and Utilization of Biological Resources in Yunnan, Kunming 650201, China.

Received: 13 September 2020 Accepted: 8 December 2020 Published online: 17 December 2020

\section{References}

Aragona M, Valente MT (2015) Genetic transformation of the tomato pathogen Pyrenochaeta lycopersici allowed gene knockout using a split-marker approach. Curr Genet 61:1-10

Chang MH, Chae KS, Han DM (2004) The GanB Ga-protein negatively regulates asexual conidiation and plays a positive role in conidial germination in Aspergillus nidulans. Genetics 167:1305-1315

Dennis C, Webster J (1971) Antagonistic properties of species groups of Trichoderma: 1. Production of non volatile metabolites. Trans Br Mycol Soc 57:25-39

do Nascimento Silva R, Steindorff AS, Ulhoa CJ, Félix CR (2009) Involvement of G-alpha protein GNA3 in production of cell wall-degrading enzymes by Trichoderma reesei (Hypocrea jecorina) during mycoparasitism against Pythium ultimum. Biotechnol Lett 31:531-536

Dubey MK, Jensen DF, Karlsson M (2014) Hydrophobins are required for conidial hydrophobicity and plant root colonization in the fungal 
biocontrol agent Clonostachys rosea. BMC Microbiol 14:18. https://doi. org/10.1186/1471-2180-14-18

Espino-rammer L, Ribitsch D, Przylucka A, Marold A, Greimel KJ, Acero H (2013) Two novel class II hydrophobins from Trichoderma spp. stimulate enzymatic hydrolysis of poly (ethylene terephthalate) when expressed as fusion proteins. Appl Environ Microbiol 79:4230-4238

Fernandes KF, Cortijotriviño D, Batista KA, Ulhoa CJ, Garcíaruiz PA (2013) Chitin hydrolysis assisted by cell wall degrading enzymes immobilized of Trichoderma asperellum on totally cinnamoylated d-sorbitol beads. Mat Sci Eng C 33:3077-3081

García-Rico RO, Gil-Duran C, Rojas-Aedo JF, Vaca I, Figueroa L, Levican G, Chávez R (2017) Heterotrimeric G protein alpha subunit controls growth, stress response, extracellular protease activity, and cyclopiazonic acid production in Penicillium camemberti. Fungal Biol 121:754-762

Guo L, Yang Y, Yang L, Wang F, Wang G, Huang J (2016) Functional analysis of the G-protein a subunits FGA1 and FGA3 in the banana pathogen Fusarium oxysporum f. sp. cubense. Physiol Mol Plant Pathol 94:75-82

Hu Y, Liu G, Li Z, Qin Y, Qu Y, Song X (2013) G protein-cAMP signaling pathway mediated by PGA3 plays different roles in regulating the expressions of amylases and cellulases in Penicillium decumbens. Fungal Genet Biol 59:62-70

Huang Y, Mijiti G, Wang Z, Yu W, Fan H, Zhang R, Liu Z (2015) Functional analysis of the class II hydrophobin gene HFB2-6 from the biocontrol agent Trichoderma asperellum ACCC30536. Microbiol Res 171:8-20

Jiang H, ZhangL ZJZ, Ojaghian MR, Hyde KD (2016) Antagonistic interaction between Trichoderma asperellum and Phytophthora capsici in vitro. J Zhejiang Univ Sci B 17:271-281

Khalesi M, Gebruers K, Derdelinckx G (2015) Recent advances in fungal hydrophobin towards using in industry. Protein J 34:243-255

Lei M, Liu J, Fang Y, Shao Y, Li L, Yu J, Chen F (2019) Effects of different G-protein a-subunits on growth, development and secondary metabolism of Monascus ruber M7. Front Microbiol 10:1555. https://doi.org/10.3389/ fmicb.2019.01555

Li M, Ma G, Lian H, Su X, Tian Y, Huang W, Mei J, Jiang X (2019) The effects of Trichoderma on preventing cucumber fusarium wilt and regulating cucumber physiology. J Integr Agric 18:607-617

Lorito M, Harman GE, Hayes CK, Broadway RM, Tronsmo A, Woo SL, Pietro AD (1993) Chitinolytic enzymes produced by Trichoderma harzianum: antifungal activity of purified endochitinase and chitobiosidase. Mol Plant Pathol 83:303-307

Minenko E, Vogel RF, Niessen L (2014) Significance of the class II hydrophobin FgHyd5p for the life cycle of Fusarium graminearum. Fungal Biol 118:385-393

Mukherjee PK, Kenerley CM (2010) Regulation of morphogenesis and biocontrol properties in Trichoderma virens by a VEL. Appl Environ Microbiol 76:2345-2352

Nogueira KMV, Costa MdN, de Paula RG, Mendonça-Natividade FC, RicciAzevedo R, Silva RN (2015) Evidence of cAMP involvement in cellobiohydrolase expression and secretion by Trichoderma reesei in presence of the inducer sophorose. BMC Microbiol 15:195. https://doi.org/10.1186/s1286 6-015-0536-z
Reithner B, Brunner K, Schuhmacher R, Peissl I, Seidl V, Krska R, Zeilinger S (2005) The G protein a subunit tga1 of Trichoderma atroviride is involved in chitinase formation and differential production of antifungal metabolites. Fungal Genet Biol 42:749-760

Rocha-Ramírez V, Omero C, Chet I, Horwitz BA, Herrera-Estrella A (2002) Trichoderma atroviride G-protein a-subunit gene tga1 is involved in mycoparasitic coiling and conidiation. Eukaryot Cell 1:594-605

Sakai K, Yokota A, Kurokawa H, Wakayama M, Moriguchi M (1998) Purification and characterization of three thermostable endochitinases of a noble bacillus strain, mh-1, isolated from chitin. Appl Environ Microb 64:3397-3402

Schmoll M, Schuster A, Silva R, Kubicek CP (2009) The G-alpha protein GNA3 of Hypocrea jecorina (Anamorph Trichoderma reesei) regulates cellulase gene expression in the presence of light. Commun Integr Biol 8:410-420

Segers GC, Nuss DL (2003) Constitutively activated Ga negatively regulates virulence, reproduction and hydrophobin gene expression in the chestnut blight fungus Cryphonectria parasitica. Fungal Genet Biol 38:198-208

Shevchuk NA, Bryksin AV, Nusinovich YA, Cabello FC, Sutherland M, Ladisch S (2004) Construction of long DNA molecules using long PCR-based fusion of several fragments simultaneously. Nucleic Acids Res 32:e19. https:// doi.org/10.1093/nar/gnh014

Song N, Dai Q, Zhu B, Wu Y, Xu M, Voegele RT, Gao X, Kang Z, Huang L (2017) Ga proteins Gvm2 and Gvm3 regulate vegetative growth, asexual development, and pathogenicityon apple in Valsa mali. PLoS ONE 7:e0173141. https://doi.org/10.1371/journal.pone.0173141

Sun Q, Jiang X, Pang L, Wang L, Li M (2016) Functions of thga1 gene in Trichoderma harzianum based on transcriptome analysis. BioMed Res Int. https ://doi.org/10.1155/2016/8329513

Susanne Z, Barbara R, Valeria S, Isabel P, Matteo L, Robert LM (2005) Signal transduction by Tga3, a novel $\mathrm{G}$ protein subunit of Trichoderma atroviride. Appl Environ Microb 71:1591-1597

Wang X, Zhang H, Pan Y, Xu J, Xu J, Feng J (2014) Effective universal vectors building for gene knockout and fluorescent expression in Fusarium graminearum. Plant Protect 40:106-112

Yu HY, Seo JA, Kim JE, Han KH, Shim WB, Yun SH, Lee YW (2008) Functional analyses of heterotrimeric $\mathrm{G}$ protein $\mathrm{G}$ alpha and $\mathrm{G}$ beta subunits in Gibberella zeae. Microbiol 154:392-401

Zeilinger S, Reithner B, Scala V, PeissI I, Lorito M, Mach RL (2005) Signal transduction by Tga3, a novel $\mathrm{G}$ protein a subunit of Trichoderma atroviride. Appl Environ Microb 71:1591-1597

Zhang S, Yu X, Kim B, Nemat OK (2011) Two hydrophobins are involved in fungal spore coat rodlet layer assembly and each play distinct roles in surface interactions, development and pathogenesis in the entomopathogenic fungus, Beauveria bassiana. Mol Microbiol 80:811-826

\section{Publisher's Note}

Springer Nature remains neutral with regard to jurisdictional claims in published maps and institutional affiliations.

\section{Submit your manuscript to a SpringerOpen ${ }^{\circ}$ journal and benefit from:}

- Convenient online submission

- Rigorous peer review

- Open access: articles freely available online

- High visibility within the field

- Retaining the copyright to your article

Submit your next manuscript at $\boldsymbol{\Delta}$ springeropen.com 